\title{
EchoGéo
}

19 | 2012

Du littoral à la haute mer : quelles recherches récentes en géographie?

\section{Entretien avec Linda Boukhris}

Maria Gravari-Barbas et Sébastien Jacquot

\author{
CpenEdition \\ Journals \\ Édition électronique \\ URL : https://journals.openedition.org/echogeo/13001 \\ DOI : 10.4000/echogeo. 13001 \\ ISSN : 1963-1197 \\ Éditeur \\ Pôle de recherche pour l'organisation et la diffusion de l'information géographique (CNRS UMR 8586) \\ Référence électronique \\ Maria Gravari-Barbas et Sébastien Jacquot, «Entretien avec Linda Boukhris », EchoGéo [En ligne], 19 | \\ 2012, mis en ligne le 10 février 2012, consulté le 10 août 2021. URL : http://journals.openedition.org/ \\ echogeo/13001; DOI : https://doi.org/10.4000/echogeo.13001
}

Ce document a été généré automatiquement le 10 août 2021.

EchoGéo est mis à disposition selon les termes de la licence Creative Commons Attribution - Pas d'Utilisation Commerciale - Pas de Modification 4.0 International (CC BY-NC-ND) 


\title{
Entretien avec Linda Boukhris
}

\author{
Maria Gravari-Barbas et Sébastien Jacquot
}

1 Linda Boukhris est Doctorante en géographie, EIREST - Université Paris 1 Panthéon Sorbonne et consultante dans un cabinet de conseil en management.

2 - Maria Gravari-Barbas (MGB), Sébastien Jacquot (SJ). Quel a été votre parcours de géographe, avant le début de votre thèse?

3 - Linda Boukhris (LB). Après un baccalauréat littéraire, j’ai réalisé trois années de classe préparatoire littéraire au Lycée Lakanal à Sceaux (hypokhâgne, khâgne, cube) dans le but de préparer le concours de l'École Normale Supérieure de Lettres et Sciences Humaines, en option Histoire-Géographie. Depuis le CM2, j'ai toujours souhaité enseigner l'histoire, une discipline que j'affectionnais tout particulièrement. L'idée de transmettre un savoir m'a très tôt enthousiasmé et a dès lors animé mon parcours universitaire. Toutefois, lorsque j'étais en classe préparatoire, j’ai réellement découvert une nouvelle discipline, qu'est la géographie, une discipline qui n'avait plus seulement le statut de petite sœur de l'histoire -statut si spécifique à la tradition académique française au demeurant. Je me suis initiée à la cartographie à travers les commentaires de cartes topographiques au $1 / 50000^{\mathrm{e}}$ des régions de France métropolitaine et d'Outremer et j'étais fascinée par la lecture si riche que l'on pouvait en faire et en quoi cela nous permettait de comprendre les relations entre l'homme et son territoire. La géographie a ainsi pris toute sa réelle dimension à mes yeux : une discipline intégrant pleinement les réalités du terrain et une discipline au cœur des grands enjeux et débats menés au sein des Sciences Sociales. La géographie est en effet une discipline "hybride" offrant les possibilités d'une stimulation intellectuelle sans spéculation aucune : afin d'expliquer les phénomènes observés et discutés, les géographes sont conduits à invoquer différents types de facteurs, qu'ils soient économique, politique, historique, sociologique, culturel, démographique... Aussi devons-nous mobiliser des savoirs multiples et de cette pluridisciplinarité naît la spécificité du géographe qui opère une synthèse des approches. Ainsi de cette affinité prononcée pour la discipline géographique et suite à une sous-admissibilité au concours de l'ENS, j'ai décidé de poursuivre un Master de Géographie à l'Université Paris IV-La Sorbonne portant sur les dynamiques spatiales des pays du Sud et les enjeux de la mondialisation. 
représentations mythifiées de la nature sont à la base de l'identité touristique du Costa Rica. Nous relevons cependant un paradoxe puisqu'au moment même où ces images sont largement diffusées par le "système touristique ", le Costa Rica connaît des taux de déforestation élevés ainsi qu'une grave crise économique et sociale, qui remet en question son modèle de stabilité. Aujourd'hui encore, la progression d'un modèle touristique balnéaire, consommateur de ressources, ainsi que le rôle croissant du costa Rica au sein de la géographie régionale du narcotrafic, ne se présentent pas comme des obstacles à la promotion de ces représentations. Le pouvoir des images est tel qu'il nie toute réalité qui contredirait l'imaginaire touristique, qui se veut également désir touristique. Parce qu'il est désir, l'imaginaire touristique est au-delà de l'intellectuelle représentation, il comprend toutes sortes d'images, de symboles, de mythes porteurs de sens, dessinant ainsi une poétique de l'espace. Concernant la première représentation mentionnée, l'imaginaire touristique reprend les caractéristiques de l'imaginaire national costaricien, construit par les élites politiques et intellectuelles du pays dès le $\mathrm{XIX}^{\mathrm{e}}$ et basé sur la « différence » costaricienne en matière de stabilité et d'exemplarité politique. Si l'imaginaire touristique contribue à amplifier la portée de l'imaginaire national costaricien dans le cadre de la mondialisation touristique, il est également producteur d'une nouvelle caractéristique, à savoir la « différence » verte. C'est en effet la puissance de l'imaginaire touristique qui a contribué à faire du Costa Rica la première destination écotouristique de l'isthme centroaméricain, produisant et diffusant l'image d'une nation toute entière dédiée à la cause verte. Si cet imaginaire touristique est à l'origine de la production des lieux touristiques, je fais l'hypothèse qu'il participe également de la construction de l'identité territoriale du Costa Rica, en produisant des symboles territoriaux à partir desquels se cristallise l'identification collective nationale.

7 L'objet de ma thèse est ainsi d'identifier, à travers le cas costaricien, les principales composantes de l'imaginaire touristique et d'analyser l'impact de cet imaginaire dans la production matérielle et symbolique de l'espace, du territoire et des hauts-lieux touristiques. Il s'agit ainsi de démontrer en quoi l'imaginaire touristique est un imaginaire instituant, producteur de territorialité.

8 - MGB, SJ. De quelle manière vous vous êtes saisie de l'objet tourisme en tant que doctorante?

9 - LB. Le tourisme est un fascinant objet de recherche car, s'il est l'expression par excellence du monde globalisé et un phénomène à analyser en tant que tel, il est également révélateur de réalités socio-économiques et culturelles complexes. En effet, appliqué à mon aire géographique de recherche qu'est l'Amérique Latine, le tourisme devient un prisme d'observation et de lecture des réalités latino-américaines et des 
grands enjeux de ces sociétés. Nathalie Raymond, grande géographe spécialiste de la question du tourisme en Amérique Latine (Pérou, Guatemala, Mexique) ne dit pas autre chose lorsqu'elle démontre, à travers le cas guatémaltèque, que le développement touristique tel qu'il s'opère n'est que le reflet et ne fait que reproduire les structures sociales et ethniques inégalitaires passées et présentes observées dans la société.

L'étude du tourisme, telle que je la conçois, ne repose pas seulement sur l'observation et l'analyse des lieux touristiques, la nature des infrastructures ou les types de consommation, elle intègre l'idée selon laquelle le tourisme constitue un axe de lecture privilégié des sociétés dans lesquelles le développement touristique se déploie. De ce fait, la dimension politique du tourisme ne doit pas être négligée et devient un des enjeux majeurs de l'analyse, étant entendu que derrière le modèle économique choisi, se dessinent des stratégies politiques complexes de la part des acteurs locaux, nationaux, régionaux et internationaux.

11 C'est également à travers une approche pluridisciplinaire et internationale que j'ai souhaité m'approprier ce sujet. Le laboratoire de recherche dans lequel j'effectue ma thèse sous la direction de Maria Gravari-Barbas, l'Institut de Recherche et d'Etudes Supérieures sur le Tourisme (IREST), s'inscrit dans cette ligne mobilisant des chercheurs et doctorants d'horizons disciplinaires différents (anthropologie, sociologie, économie, droit, architecture, histoire...). L'idée étant de susciter une émulation intellectuelle au sein de l'équipe de recherche, confrontée à des approches et des méthodologies différentes mais travaillant autour d'un seul et même objet de recherche qu'est le tourisme. C'est pourquoi, les références mobilisées afin de définir mon cadre conceptuel proviennent de champs disciplinaires variés tels que l'anthropologie ou la philosophie afin de caractériser au mieux l'imaginaire touristique. L'approche internationale s'illustre quant à elle à travers mes multiples missions de terrain au Costa Rica, où l'un des objectifs assignés est d'appréhender la littérature scientifique sur la question du tourisme, ou encore à l'occasion d'un séjour de recherche à l'Université de Berkeley (Californie). A ce titre, un groupe de chercheurs travaillant sur le tourisme (le Tourism Studies Working Group -TSWG) s'est mis en place au sein du Département d'Anthropologie de UC Berkeley avec les mêmes objectifs qu'énoncés précédemment pour l'IREST. Je me suis rendue à une de leurs conférences co-organisée avec l'Université Paris I sur les imaginaires touristiques, permettant ainsi de multiplier les rencontres et les échanges interdisciplinaires et interculturels en lien avec mon sujet.

12 - MGB, SJ. De quelle manière la pensée géographique structure-t-elle votre approche?

13 - LB. La pensée géographique est extrêmement présente dans mon approche dans la mesure où le territoire -objet géographique par excellence- demeure le pivot central de ma réflexion. Il s'agit pour moi de démontrer dans quelle mesure les représentations et les discours, articulés autour de la notion d'imaginaire touristique, ont le pouvoir de façonner des pratiques spatiales et de produire de la territorialité. En d'autres termes, et de façon quelque peu schématique, de quelle manière le tourisme façonne le territoire. En effet, l'imaginaire touristique, fer de lance du processus de qualification du territoire au Costa Rica (quasi $1 / 3$ du territoire national protégé), garantit l'inscription de la nation costaricienne dans un lieu valorisé et valorisant et contribue à spatialiser, matérialiser une identité. L'imaginaire touristique est ainsi au cœur du processus d'appropriation et de marquage de l'espace. Et c'est précisément ce processus que je tente d'appréhender à travers mon sujet. Quant à la littérature 
mobilisée, si elle se veut pluridisciplinaire, elle ne peut faire l'économie des grands penseurs de la géographie contemporaine francophone et anglophone.

14 - MGB, SJ. Comment établissez-vous des passerelles entre votre emploi et la recherche, en termes de méthodes, ...?

15 - LB. Des passerelles ont pu être établies à plusieurs égards entre mon emploi et la recherche.

16 En effet, en matière de méthodologie, cela m'a permis d'acquérir de façon plus aboutie et de maîtriser de façon durable les techniques d'entretiens, qui sont un des outils majeurs de collecte de données sur mon terrain. De fait, lorsque je me rends au Costa Rica, je suis tenue de définir un organigramme des acteurs clés du développement touristique aussi bien à l'échelle nationale qu'au niveau de mes deux études de cas locales et de réaliser un certain nombre d'entretiens avec des acteurs aussi multiples que les institutions publiques ou privés (directeurs de départements du Ministère du Tourisme, président de la Chambre Nationale du Tourisme, membres du Ministère de l'Environnement et du Système de Protection Nationale...) mais également les acteurs du secteur professionnel (les propriétaires d'hôtels, de tours opérateurs, de restaurants...), les associations locales et les touristes bien entendu...

17 Le professionnalisme et la maîtrise des "soft skills", que j'ai pu acquérir au cours de mon activité professionnelle constituent également des atouts à déployer afin de permettre une collecte de données des plus productives. En effet, sur le terrain, obtenir de l'information peut passer par la réalisation de stages au sein de structures aussi diverses que le Ministère du Tourisme, une agence de voyages ou une association de développement locale. Etudier le phénomène touristique nécessite une grande capacité d'adaptation car l'un des enjeux de la recherche est précisément d'intégrer l'ensemble des discours des multiples acteurs impliqués dans le système.

18 Je pourrais également mentionner la maîtrise des outils de management de projet qui se révèle fort utile au sein de laboratoires où les travaux de recherche sont de plus en plus associés à la constitution de dossiers (appels d'offre, appels à communication ou autres...). L'idée étant de rationnaliser les tâches administratives, à travers une organisation optimisée, afin de dégager du temps pour la recherche et l'enseignement. 